group of patients with and without NPS were $5.44 \pm 2.03,3.45 \pm 1.82$; respectively $(P<0.001)$. Pain was most frequently seen in wrist-hand $(50.6 \%)$ and ankle-foot (43.4\%) regions; albeit, NPS rates were highest in face $(94.4 \%)$, lower leg $(87.5 \%)$ and gluteal $(78.6 \%)$ regions. SF 36 scores were lower in patients with NPS than the patients without NPS but the difference has not reached to a statistically significant level $(P>0.05)$. The most associated factors with NPS were Medsger Disease Severity Score for muscle and drug consumption of the patient.

Conclusions: According to our results, high frequency of NPS is seen in SSc patients, and NPS is associated with low QoL. The highest rates of NPS presence were seen in face, gluteal and lower leg regions of the body. Differential diagnosis of NPS is important to consider right treatment options and accurate management of pain in all rheumatologic diseases including SSc.

Disclosure of Interest: None declared

DOI: 10.1136/annrheumdis-2017-eular.6213

\section{SAT0356 VIDEOFLUOROSCOPY SWALLOW STUDY IN PATIENTS WITH SYSTEMIC SCLEROSIS. CORRELATION WITH CLINICAL PATTERNS}

P. Fraticelli ${ }^{1}$, C. Fischetti ${ }^{2}$, A.M. Pisani ${ }^{3}$, S. Barchiesi ${ }^{2}$, G. Romanelli ${ }^{2}$, A. Gabrielli ${ }^{1}, 2 .{ }^{1}$ Clinica medica, Department of internal medicine, Ospedali Riuniti; ${ }^{2}$ Department of Clinical and Molecular Sciences, Università Politecnica delle Marche; ${ }^{3}$ Department of Radiology, Ospedali Riuniti, Ancona, Italy

Background: Systemic Sclerosis (SSc) is a chronic autoimmune disease characterized by proliferative vascular lesions and progressive fibrosis of skin and internal organs, including the gastrointestinal tract. Gastrointestinal involvement is a very frequent complication, reported in up to $90 \%$ of SSc patients in both limited (ISSc) and diffuse (dSSc) cutaneous forms, and it is one of the earliest events.

Objectives: To evaluate the correlation between radiological items analyzed by videofluoroscopy swallow study and clinical patterns of patients SSc.

Methods: 55 patients (M/F: 6/49; median age 56y; median disease $6 y$, ISSc /dSSc:36/19; anti-Scl70+:21/55, ACA+:18/55, only ANA+:16/55), with a diagnosis of SSc and a history of dysphagia underwent a dynamic and morphological study of the oral, pharyngeal and esophageal phases of swallowing with videofluoroscopy. The oral and pharyngeal esophageal phases were performed in the upright position, while the esophageal phase was performed in the proneoblique position, after administration of contrast material either in bolus form or diluted. Data were analyzed by radiologist with experience in videofluoroscopy for the evaluation of 17 videofluoroscopy items, of which, 4 concerning the oral, 4 the pharyngeal and 9 the esophageal phase, respectively. Results were expressed in a binary system. Then the main relevant videofluoroscopy findings were correlated with the principal scleroderma pattern of disease: ISSc vs dSSc; disease duration (more than 2 years) and subset of autoantibodies.

Results: Radiological study of swallowing disorders showed for oral phase: inadequate velar elevation in $4 \%$, leakage in $15 \%$, drooling in none $(0 \%)$ and stasis of bolus in mouth in $4 \%$ of the patients. As for pharyngeal phase: stasis of bolus on pharyngeal in $49 \%$, penetration in the laryngeal aditus in $53 \%$, postswallowing aspiration in $22 \%$, abnormal upper esophageal sphincter behavior in $13 \%$ of the cases. Concerning esophageal phase: inadequate primary peristalsis in $53 \%$, abnormal secondary peristalsis in $29 \%$, non-peristaltic contractions in $40 \%$, defects of clearance in $69 \%$, abnormal lower esophageal sphincter behavior in $76 \%$, hiatal hernia in $80 \%$, esophageal reflux in $56 \%$, esophagitis in $82 \%$ of the patients, nobody presented esophageal luminal stenosis. When we analyzed the swallowing disorders in different conditions we found that these are prevalent in patients with more than 2 year of disease, although may be found also early. Conversely, we have not found a significant prevalence between the ISSc or the dSSc, or a particular correlation with different patterns of autoantibodies.

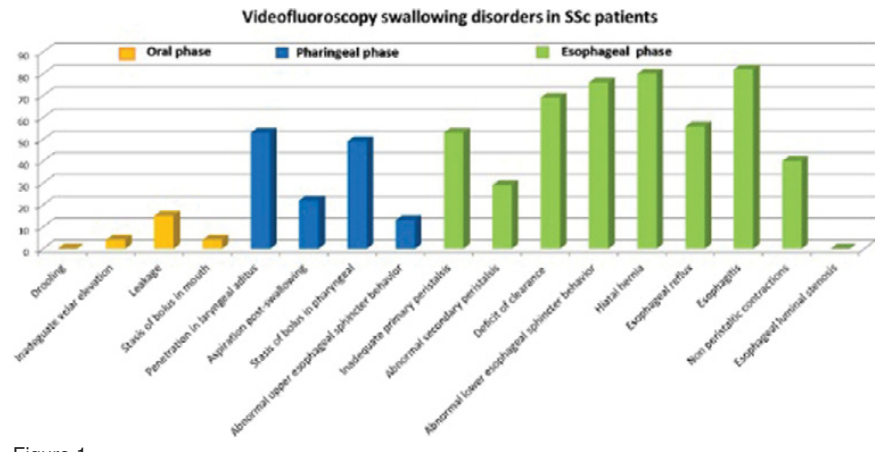

Figure 1

Conclusions: Our study demonstrated relevant abnormalities in swallowing functions in high number of patients with SSc. Pharyngeal and esophageal phases are the most affected, also early. Swallowing disorders increase with disease progression and involve similarly the limited or the diffuse SSc. An early and detailed diagnosis, supported by a semi-quantitative analysis with the use of videofluoroscopy scores, may be useful to guide the appropriate therapeutic approach, either rehabilitative or pharmacological, and finally, to improve the patient's quality of life. Extensive studies are necessary to confirm and transfer our data into clinical practice.

Disclosure of Interest: None declared

DOI: 10.1136/annrheumdis-2017-eular.5456

\section{SAT0357 FEATURES ASSOCIATED WITH MODERATE TO HIGH RISK OF MALNUTRITION IN A COHORT OF PATIENTS WITH SYSTEMIC SCLEROSIS}

P.M. Marcaida ${ }^{1}$, D. Vanesa ${ }^{1}$, V. Martire ${ }^{1}$, F. Melo $^{1}$, A. Secco ${ }^{1}$, M. Mamani ${ }^{1}$, S. Scarafia ${ }^{1}$, M. Lazaro ${ }^{2}$, A. Cusa ${ }^{2}$, F. Cusa $^{3}$, V. Caputo ${ }^{4}$, J. Sarano ${ }^{5}$, M. Molina ${ }^{6}$, A. Nitsche ${ }^{7}$, M. Gaona ${ }^{8}$, C. Pena ${ }^{9}$, M. Garcia ${ }^{9}$, S. Gordon ${ }^{10}$, N. Muñoz Cantos ${ }^{8}$, S. Montoya ${ }^{8} .{ }^{1}$ Rheumatology, Rivadavia Hospital;

${ }^{2}$ Rheumatology; ${ }^{3}$ Nutrition, IARI; ${ }^{4}$ Rheumatology, Military Hospital;

${ }^{5}$ Rheumatology, Lanari Institute; ${ }^{6}$ Rheumatology, Central Hospital of San Isidro; ${ }^{7}$ Rheumatology, Diagnostic system-CM; ${ }^{8}$ Rheumatology, Ramos Mejia Hospital, Buenos Aires; ${ }^{9}$ Rheumatology, HIGA, la plata; ${ }^{10}$ Rheumatology, HIGA, Mar del plata, Argentina

Background: It is estimated that about $28 \%$ of Systemic Sclerosis (SS) patients have moderate to high risk of malnutrition.

Objectives: to evaluate differences between SS patients with moderate to high risk of malnutrition and those with low risk.

Methods: cross-sectional, observational, multicentric study. We included patients with SS according to ACR-EULAR 2013 classification criteria. Patients were classified in groups depending on whether they were in low or moderate-high risk of malnutrition, according to the screening method for detection of adult malnutrition (MUST). Were evaluated: disease duration, disease subtype (limited or diffuse), presence of microstomia, xerostomia, active or past digital ulcers, amputations, arthritis, Rodnan Score, gastroesophageal and bowel involvement, anxiety and depression, and hands functionality by Duruöz Index. Continuous variables were described as median (IQR) or mean (SD) and percentages for categorical variables. Mann Whitney or t-test was used for continuous variables, and Fisher exact test or chi squared for categorical variables. A $p<0.05$ was considered significant. A multivariate analysis was made taking MUST as a dependent variable.

Results: 116 patients were included. Thirty percent were at moderate to high risk of malnutrition. These patients experienced significantly higher frequency of diffuse SS (49\% vs $21 \%, p=0,003)$, bowel involvement $(49 \%$ vs $27 \%$. $p=0,02)$, gastroesophageal involvement $(74 \%$ vs $48 \%, p=0,009)$, higher cutaneous involvement (median 12 vs $6, p=<0,01$ ), microstomia ( $40 \%$ vs $15 \%, p=0,003$ ), worst hand functionality (median: 11 vs $3, p=0,02$ ), and moderate-severe depression $(37 \%$ vs $16 \%, p=0,012)$. Also, men experienced a higher moderatehigh risk of malnutrition $(20 \%$ vs $6 \%, p=0,02)$. In the multivariate analysis, the male sex (OR 4.55, 95\% Cl 1.11-20, $\mathrm{p}=0.03$ ), the Rodnan score $>9$ (OR 3.13 , $95 \% \mathrm{Cl}, 95 \% \mathrm{Cl}, \mathrm{p}=0,01$ ), and gastroesophageal involvement (OR 2.87, 95\% Cl $1.07-7.73, p=0.03$ ), were independently and statistically significant.

Conclusions: These results highlight the importance of assessing the nutritional status of our SS patients.

Disclosure of Interest: None declared

DOI: 10.1136/annrheumdis-2017-eular.6535

\section{SAT0358 DECREASED BODY FAT, LEAN BODY MASS AND BONE MINERAL DENSITY IN PATIENTS WITH SYSTEMIC SCLEROSIS ARE ASSOCIATED WITH DISEASE ACTIVITY AND PHYSICAL ACTIVITY}

S. Oreska $^{1}$, M. Spiritovic ${ }^{1,2}$, P. Cesak ${ }^{2}$, M. Cesak ${ }^{2}$, H. Storkanova ${ }^{1}$, K. Kubinova ${ }^{1}$, M. Klein ${ }^{1}$, L. Vernerova ${ }^{1}$, O. Ruzickova ${ }^{1}$, H. Mann ${ }^{1}$, K. Pavelka ${ }^{1}$ L. Senolt ${ }^{1}$, J. Vencovsky ${ }^{1}$, R. Becvar ${ }^{1}$, M. Tomcik ${ }^{1} .{ }^{1}$ Department of Rheumatology, 1st Medical Faculty, Charles University, Institute of Rheumatology; ${ }^{2}$ Faculty of Physical Education and Sport, Charles University, Prague, Czech Republic

Background: Systemic sclerosis (SSc) is characterized by fibrosis of the skin and visceral organs, especially digestive tract, and musculoskeletal involvement, which limit mobility/self-sufficiency of patients, and can have a negative impact on body composition.

Objectives: To assess body composition and physical activity of SSc patients and healthy controls $(\mathrm{HC})$

Methods: 59 patients with SSc (50 females, 9 males; mean age 52.1; disease duration 6.7 years; limited cutaneous (IcSSc,36)/diffuse cutaneous (dcSSc,23)) and 36 age-/sex-matched $\mathrm{HC}$ (30 females, 6 males, mean age 51.4) without rheumatic/tumor diseases or manifest cardiovascular event were included. SSc patients fulfilled EULAR/ACR 2013 criteria. Anthropometric parameters and body composition were assessed (by densitometry-iDXA Lunar, and by bioelectric impedance-BIA-2000-M), and physical activity was evaluated using Human Activity Profile (HAP) questionnaire. Routine biochemistry analysis was performed after 8 hours of fasting. Disease activity was evaluated by EUSTAR SSc activity score. Data are presented as mean $\pm S D$.

Results: Compared to $\mathrm{HC}$, patients with SSc had significantly lower body-mass index (BMI: $26.4 \pm 3.3$ vs. $22.4 \pm 4.3 \mathrm{~kg} / \mathrm{m}^{2}, \mathrm{p}<0.0001$ ) and body fat $\%$ assessed by both iDXA (BF\%: $37.2 \pm 6.6$ vs. $32.6 \pm 8.2 \%, p=0.0014)$ and BIA (BF\%: $31.1 \pm 6.4$ vs. 
$24.6 \pm 7.8 \%, p<0.0001)$, and a trend to decreased visceral fat weight $(0.9 \pm 0.9$ vs. $0.5 \pm 0.5 \mathrm{~kg}, \mathrm{p}=0.0670$ ). Compared to HC, SSc patients demonstrated significantly decreased lean body mass assessed by both iDXA (LBM: $46.6 \pm 7.5$ vs. $40.9 \pm 6.8 \mathrm{~kg}$, $\mathrm{p}=0.0003$ ) and BIA (LBM: $53.2 \pm 8.7$ vs. $47.7 \pm 7.0 \mathrm{~kg}, \mathrm{p}=0.0017$ ), and increased $\mathrm{ECM} / \mathrm{BCM}$ ratio (extracellular mass/body cell mass: $1.03 \pm 0.1$ vs. $1.29 \pm 0.4$, $\mathrm{p}<0.0001$ ), which reflects worse muscle predispositions for physical exercise, aerobic fitness/performance, and usually increases with deteriorating nutritional status. Compared to HC, SSc patients had significantly lower bone mineral density (BMD: $1.16 \pm 0.10$ vs. $1.05 \pm 0.11 \mathrm{~g} / \mathrm{cm}^{2}, p<0.0001$ ), and were currently able to perform less energetically demanding physical activities according to HAP score ( $84.7 \pm 6.6$ vs. $64.1 \pm 17.2, p<0.0001)$. Disease activity negatively correlated with $\mathrm{BF} \%(\mathrm{r}=-0.324, \mathrm{p}=0.014)$, and physical activity (HAP) positively correlated with $\operatorname{BMD}(r=0.276, p=0.034)$ and negatively with $\mathrm{ECM} / \mathrm{BCM}(\mathrm{r}=-0.625, \mathrm{p}<0.0001)$.

Conclusions: Compared to healthy age-/sex-matched individuals we found significant negative changes in body composition of our SSc patients, which are associated with their disease activity and physical activity, and could reflect their nutritional status, and gastrointestinal and musculoskeletal involvement.

Acknowledgements: Supported by AZV-16-33574A, GAUK-214615.

Disclosure of Interest: None declared

DOI: 10.1136/annrheumdis-2017-eular.4873

\section{SAT0359 THE COURSE OF MOUTH OPENING AND ITS RELATIONSHIP WITH DISEASE CHARACTERISTICS, GLOBAL FUNCTIONING, HEALTH-RELATED QUALITY OF LIFE AND MOUTH HANDICAP IN PATIENTS WITH SYSTEMIC SCLEROSIS}

S.J.H. Khidir ${ }^{1}$, M. Boonstra ${ }^{1}$, G.W.M. Boerrigter ${ }^{2}$, E.M. Voogt-van der Harst ${ }^{2}$, S.A. Bergstra ${ }^{1}$, M.K. Ninaber ${ }^{3}$, N. Ajmone Marsan ${ }^{4}$, T.W.J. Huizinga ${ }^{1}$, J.K. de Vries-Bouwstra ${ }^{1} .{ }^{1}$ Rheumatology; ${ }^{2}$ Physiotherapy; ${ }^{3}$ Pulmonology; ${ }^{4}$ Cardiology, Leiden University Medical Center, Leiden, Netherlands

Background: Systemic sclerosis (SSc) is a fibrotic disease which can lead to reduced maximal mouth opening (MMO). Previous cross-sectional research showed that reduced MMO in SSc correlated with higher disease severity and lower oral quality of life. Interpretation of interventions that possibly influence $\mathrm{MMO}$ is difficult, as the natural history of MMO in SSc is not well described.

Objectives: To evaluate in SSc 1) the course of MMO 2) disease characteristics predictive for decreasing $\mathrm{MMO}$ and 3) the relationship between the course of MMO and global functioning, health-related quality of life (HRQoL) and mouth handicap.

Methods: SSc patients from the Leiden Combined Care In Systemic Sclerosis (CCIS) cohort, Leiden University Medical Center were included if at least one MMO measurement was available. Annual clinical assessment includes MMO

Table 1. Multivariate mixed model to determine the association between Maximal Mouth Opening (MMO) and baseline disease characteristics in SSc patients

\begin{tabular}{|c|c|c|c|c|}
\hline & \multirow[t]{2}{*}{$\beta$} & \multirow[t]{2}{*}{$p$-value } & \multicolumn{2}{|c|}{$95 \%$ Confidence Interval } \\
\hline & & & Lower limit & Upper limit \\
\hline Non cutaneous SSc (3) & & & ref & \\
\hline Limited SSc (2) & -2.2 & 0.036 & -4.2 & -0.2 \\
\hline Diffuse SSc (1) & -4.1 & 0.004 & -6.9 & -1.4 \\
\hline Gastrointestinal involvement & -2.6 & 0.001 & -4.1 & -1.1 \\
\hline Pulmonary involvement & -1.8 & 0.032 & -3.4 & -0.2 \\
\hline Peripheral vasculopathy & -2.3 & 0.003 & -3.8 & -0.8 \\
\hline
\end{tabular}

Figure 1 Mean Maximal Mouth Opening (MMO), percentage of microstomia and change in MMO over time in SSc patients

Mean MMO and percentage of microstomia over time

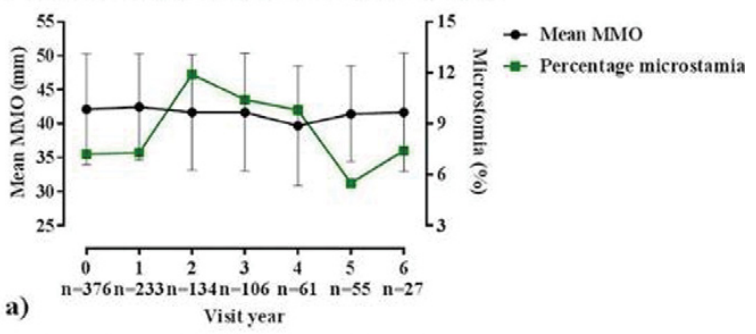

Change in MMO between consecutive visits

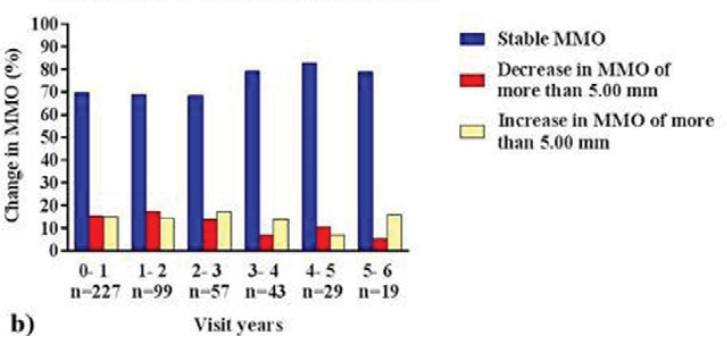

measurement, global functioning (HAQ), HRQoL (Short Form-36; SF-36), mouth handicap (Mouth Handicap in Systemic Sclerosis scale; MHISS). We assessed mean $\mathrm{MMO}$, prevalence of microstomia $(\mathrm{MMO}<30.00 \mathrm{~mm})$ and decreasing $\mathrm{MMO}$ (decline $>5.00 \mathrm{~mm} /$ year) over time. Predictors for decreasing $\mathrm{MMO}$ were assessed by a linear mixed model (LMM), including baseline clinical parameters reflecting 7 domains (peripheral vasculopathy, skin, heart, gastrointestinal, lung, renal, musculoskeletal). Additionally, MMO over time was correlated with baseline HAQ, SF-36 and MHISS in separate LMMs adjusted for gender, Body Mass Index, age and disease characteristics correlated significantly $(p<0.05)$ with the course of MMO.

Results: 382 patients were included with mean age $54 \pm 14$ years, $83 \%$ female and $25 \%$ diffuse cutaneous SSc. Mean MMO during 6 years of follow-up ranged from 39.4 to $42.5 \mathrm{~mm}$. The annual mean percentage of patients with microstomia was $9 \%$, range 6 to $12 \%$ (Figure 1a). A decrease in MMO between two consecutive annual measurements was observed in 5 to $17 \%$ of patients, mean $12 \%$ (Figure $1 b)$. More extended cutaneous involvement, peripheral vasculopathy, pulmonary and gastrointestinal involvement at baseline were predictive for decreasing MMO over time (Table 1). Baseline HAQ ( $\beta=-1.6,95 \% \mathrm{Cl}=-2.7$ to -0.6$)$, SF-36 physical component $(\beta=0.1,95 \% \mathrm{Cl}=0.0$ to 0.1$)$ and $\mathrm{MHISS}(\beta=-0.2,95 \% \mathrm{Cl}=-0.2$ to -0.1$)$ correlated with longitudinal MMO.

Conclusions: Over time, MMO is relatively stable in the majority of SSc patients. Microstomia is seen in $9 \%$ and important decrease of MMO in $12 \%$ and is associated with more severe organ involvement. Even though this concerns only a small subgroup of SSc patients, a significant association with global functioning and HRQoL was demonstrated, underlining the need for treatment strategies improving MMO.

Disclosure of Interest: None declared

DOI: 10.1136/annrheumdis-2017-eular.6245

\section{SAT0360 MYOSITIS-SPECIFIC AND MYOSITIS-ASSOCIATED AUTOANTIBODIES IN PATIENT WITH DERMATOMYOSITIS / POLYMYOSITIS; COMPARISON BETWEEN LINE BLOT AND ENZYME-IMMUNOASSAY ASSAYS}

S. Nakashima, T. Kameda, M. Izumikawa, H. Shimada, H. Ozaki, R. Wakiya, A. Kondou, N. Kadowaki, H. Dobashi. Department of Internal medicine, Division of Hematology, Rheumatology and Respiratory Medicine, Kagawa University, Kagawa, Japan

Background: MESACUPTM test (enzyme-immunoassay assays; TIF1 $\gamma$, MDA5, Jo-1, EJ, PL-7, PL-12, and KS; MBL) (MESA) is used for a diagnosis of idiopathic inflammatory myopathies (IIMs) in Japan. On the other hand, EUROLINE myositis Profile 3 (line blot; EUROIMMUN) (EURO) can analyze plural Myositis-specific autoantibodies (MSA: Mi-2, Jo-1, SRP, PL-7, PL-12, EJ, OJ) and Myositisassociated autoantibodies (MAA; Ku, PM-Scl) at the same time, which is used commercially in Western countries. However, the difference between utility of MESA and that of EURO haven't be disclosed.

Objectives: To clarify difference between utility of EURO and MESA, and extract the problem of respective examination.

Methods: We enrolled 58 patients diagnosed DM/PM in our facility. Polymyositis (PM) and dermatomyositis (DM) were diagnosed according to Bohan and Peter's criteria. ${ }^{1)}$ The MAA and MSA were analyzed using MESA and EURO. In case of MESA (+), MSA (anti-Jo1, anti-PL7, anti-PL12, anti-KS) were identified by specific ELISA. When those results were different, we analyzed by immunoprecipitation. And we analyzed the association between each autoantibody and clinical features. Results: MSA and MAA were detected in 43/58 (74\%) (anti-PL7: 12, anti-Jo1: 7 , anti-EJ: 3, anti-PL12: 1, anti-OJ: 0, anti-Ro52: 27, anti-PM-Scl75: 7, anti-Ku: 6, anti-PM-Scl100: 1) by EURO. On the other hand, MSA and MAA were detected in 30/58 (52\%) (anti-PL7: 9, anti-Jo1: 7, anti-EJ: 4, TIF1 $\gamma$ : 4, MDA5: 3, U1RNP: 3) by MESA. Five patients were MESA (-) and EURO (-). In the case of ARS positive patient, Two of EURO (-) patients was positive in MESA, respectively Jo1 and EJ. Three of MESA (-) patients was positive in EURO. Although MESA(-) and $\mathrm{EURO}(+)$ patients had plural MSA and MAA (PL7+Jo1, PL7+PL12, PL7+Ku), MSA and MAA weren't detected by immunoprecipitation in MESA(-) and EURO(+) patients. Two of patients that detected plural MSA or MAA had rapid progressive ILD.

(Association between clinical manifestations and MSA, MAA)

All patients with anti-ARS (anti-Jo-1, anti-PL-7, anti-PL-12 and anti-EJ) had ILD. In addition, anti-ARS were associated with arthritis and mechanic's hands. Anti-Mi-2 positive patients didn't have ILD. Patients detected anti-PM-Scl75, anti-PM-Scl100, anti-Ku were almost overlap syndrome. All of Anti-SRP positive patients was PM.

Conclusions: EURO is a convenient and reliable method useful for detection of MSA and MAA. It was suggested the patients whom plural antibodies were detected by EURO have unique clinical course in others.

References:

[1] Bohan, A., Peter, J.B., 1975. Polymyositis and dermatomyositis (second of two parts). N. Engl. J. Med. 292, 403-407.

Disclosure of Interest: None declared

DOI: 10.1136/annrheumdis-2017-eular.4762 